\title{
Prophylactic Cefazolin in Amnioinfusions Administered for Meconium-Stained Amniotic Fluid
}

\author{
R.K. Edwards* and P. Duff \\ Department of Obstetrics and Gynecology, Division of Maternal-Fetal Medicine, University of \\ Florida College of Medicine, Gainesville, FL
}

\begin{abstract}
Objective: To determine if amnioinfusion with an antibiotic solution decreased the rate of clinical chorioamnionitis and puerperal endometritis in patients with meconium-stained amniotic fluid.

Methods: Patients in labor at 36 weeks of gestation or greater with singleton pregnancies and meconium-stained amniotic fluid were randomized to receive either cefazolin, $1 \mathrm{~g} / 1,000 \mathrm{~mL}$, of normal saline $(n=90)$ or normal saline $(n=93)$ amnioinfusion. Rates of clinically diagnosed chorioamnionitis and endometritis and of suspected and culture-proven neonatal infection were determined.

Results: Between the study and control groups, the incidences of clinical chorioamnionitis (7.8\% vs. $8.6 \%)$, endometritis $(2.4 \%$ vs. $3.5 \%)$, aggregate intrauterine infection $(10.0 \%$ vs. $11.8 \%)$, suspected neonatal infection $(17.8 \%$ vs. $21.5 \%)$, and proven neonatal infection $(0.0 \%$ vs. $2.2 \%)$ were not significantly different.

Conclusions: Prophylactic use of cefazolin in amnioinfusions did not significantly reduce rates of maternal or neonatal infection in patients with meconium-stained amniotic fluid. Infect. Dis. Obstet. Gynecol. 7:153-157, 1999. @ 1999 Wiley-Liss, Inc.
\end{abstract}

KEY WORDS

chorioamnionitis; endometritis; antibiotics; antibiotic prophylaxis

$M^{2}$ econium aspiration syndrome, an uncommon but serious neonatal complication, occurs most often in term or postterm infants who pass meconium in utero. Meconium-stained amniotic fluid (MSAF) complicates $8-16 \%$ of all deliveries. ${ }^{1}$ Though controversial in the absence of variable decelerations, ${ }^{2}$ amnioinfusion is standard treatment for patients with MSAF at our institution and others. By diluting meconium, amnioinfusion has been associated with decreased frequency of thick meconium, ${ }^{3,4}$ less meconium below the neonate's vocal cords, ${ }^{3-8}$ and a decrease in the rate of meconium aspiration syndrome. ${ }^{3,6,8}$

Meconium has also been identified as a risk fac- tor for microbial invasion of the amniotic cavity. ${ }^{7,8}$ In vitro, it has been found to enhance bacterial growth ${ }^{9,10}$ and impair immune function. ${ }^{11}$ Clinically, meconium is associated with an increased incidence of chorioamnionitis ${ }^{11-14}$ and endometritis. $^{13,14}$

One study has shown a significant reduction in the rate of clinical chorioamnionitis when intravenous ampicillin-sulbactam was administered prophylactically for the indication of MSAF. ${ }^{15}$ Since amnioinfusion is utilized in the setting of MSAF, and meconium is an identified risk factor for infection, we thought it logical to investigate the utility of antibiotics in the amnioinfusate. Additionally,

Grant sponsor: Southern Medical Association, Birmingham, Alabama.

*Correspondence to: Rodney K. Edwards, MD, University of Florida College of Medicine, Department of Obstetrics and Gynecology, PO Box 100294, Gainesville, FL 32610-0294. 
true antibiotic prophylaxis should employ an inexpensive, nontoxic agent that has reasonable activity against most pelvic pathogens. Accordingly, we designed a prospective, randomized, double-blinded, placebo-controlled trial to assess the efficacy of prophylactic cefazolin added to amnioinfusions administered for MSAF.

\section{SUBJECTS AND METHODS}

From September 11, 1996, to March 16, 1998, patients at Shands Hospital who had MSAF diagnosed after rupture of membranes were evaluated for participation. The study was approved by the University of Florida Health Center Institutional Review Board. Patients meeting study criteria were asked to participate, and, if they were willing, written informed consent was obtained.

Inclusion criteria were 1) labor, 2) singleton gestation, 3) 36 weeks or greater gestational age, 4) live fetus, and 5) MSAF deemed by the patient's physician to be of sufficient thickness to administer an amnioinfusion for the purpose of prophylaxis against meconium aspiration syndrome. Patients were excluded from enrollment if they 1) were allergic to cefazolin or had a history of an anaphylactic reaction to penicillin, 2) had been diagnosed with chorioamnionitis prior to being diagnosed with MSAF, or 3) had a contraindication to labor.

Group assignment was determined by means of a computerized random number generator. A vial of either one gram of cefazolin powder or $15 \mathrm{~mL}$ of normal saline was placed in numbered opaque envelopes by the hospital's research pharmacist. These envelopes were maintained in a central location in the labor and delivery suite. Once a subject was deemed eligible and gave consent for study participation, the physician caring for the patient provided the next envelope in sequence to the patient's nurse, who mixed the vial into one liter of normal saline. The patient, her physician, and the investigators were blinded to the patient's group assignment.

Amnioinfusions were administered according to a standard protocol used at our institution. Ambient temperature normal saline was infused through an intrauterine pressure catheter using gravity drainage. After an initial bolus dose of $200 \mathrm{~mL}$, the patient's nurse assessed fluid return, uterine tonus, and fluid character. As long as some return was seen, baseline uterine tone was below $15 \mathrm{mmHg}$, and the fluid return was not yet clear, the amnioinfusion was continued. These variables were reassessed after every $100 \mathrm{~mL}$ of amnioinfusion.

Patients requiring cesarean delivery received one gram of cefazolin intravenously at cord clamping and a second dose 8 hours later. This was due to the fact that all patients enrolled had at least one risk factor for endometritis, namely MSAF.

The primary outcome measures were the occurrence of either clinical chorioamnionitis or puerperal endometritis. The incidence of aggregate maternal infection (clinical chorioamnionitis plus endometritis) was calculated for each group. Other outcome variables were suspected neonatal infection, as evidenced by the neonate receiving intravenous ampicillin and gentamicin pending culture results, and culture-proven neonatal infection.

Clinical chorioamnionitis was diagnosed based on the presence of one or more of the following: maternal temperature of $38^{\circ} \mathrm{C}$ or greater, maternal or fetal tachycardia, uterine tenderness, or foulsmelling amniotic fluid. The diagnosis of puerperal endometritis was also made clinically in patients with temperature over $38^{\circ} \mathrm{C}$ on two occasions postpartum, uterine tenderness, and/or foul-smelling lochia in the absence of other localizing signs of infection. Patients who had clinical chorioamnionitis and persistent fever postpartum were not also considered to have endometritis.

Sample size calculations assumed a rate of aggregate maternal infection of $40 \%$ (based on incidences in the literature ${ }^{10,12,13,15,17,18}$ ) and a $50 \%$ reduction in infection rate with antibiotic prophylaxis. For a significance level of a type-I error of 0.05 and a type-II error of $0.2,93$ subjects in each study arm were necessary. Statistical analysis was performed with the use of the uncorrected chisquare and Fisher exact test for proportional data as appropriate, and the unpaired, two-tailed $t$-test was used for continuous variables. Ninety-five percent confidence intervals were calculated for discrete outcome variables.

\section{RESULTS}

Over the 18-month study period, 200 patients were enrolled and randomized, 100 each to the cefazolin and placebo groups. Seventeen patients were excluded from data analysis. Three of these patients delivered before amnioinfusion could be administered, two received intravenous antibiotics for sub- 
TABLE I. Demographic and clinical profile of study patients ${ }^{\mathrm{a}}$

\begin{tabular}{lrr}
\hline & $\begin{array}{r}\text { Cefazolin } \\
(\mathrm{n}=90)\end{array}$ & $\begin{array}{r}\text { Placebo } \\
(\mathrm{n}=93)\end{array}$ \\
\hline Age (yr) & $24.2 \pm 6.1$ & $23.7 \pm 5.7$ \\
Nulliparity & $26(28.9 \%)$ & $37(39.8 \%)$ \\
Race & & \\
$\quad$ White & $45(50.0 \%)$ & $35(37.6 \%)$ \\
$\quad$ Black & $35(38.9 \%)$ & $46(49.5 \%)$ \\
Other & $10(11.1 \%)$ & $12(12.9 \%)$ \\
Gestational age (wk) & $39.8 \pm 1.3$ & $39.5 \pm 1.2$ \\
Duration of ROM (h) & $6.7 \pm 5.6$ & $7.6 \pm 8.8$ \\
Duration of labor (h) & $13.5 \pm 6.7$ & $13.9 \pm 8.2$ \\
Thick meconium & & \\
$\quad$ (vs. moderate or unspecified) & $48(53.3 \%)$ & $47(50.5 \%)$ \\
More than 4 vaginal & & \\
$\quad$ exams after ROM & $26(28.9 \%)$ & $29(31.2 \%)$ \\
Infant's birth weight (g) & $3,432 \pm 453$ & $3,421 \pm 471$ \\
Delivery & & \\
$\quad$ Spontaneous vaginal & $63(70.0 \%)$ & $60(64.5 \%)$ \\
$\quad$ Operative vaginal & $10(11.1 \%)$ & $14(15.1 \%)$ \\
$\quad$ Cesarean & $17(18.9 \%)$ & $19(20.4 \%)$ \\
GBS prophylaxis & $21(23.3 \%)$ & $22(23.7 \%)$ \\
\hline
\end{tabular}

${ }^{a}$ ROM, rupture of membranes. Data are presented as $n(\%)$ or mean \pm standard deviation. None of the observed differences were statistically significant.

acute bacterial endocarditis prophylaxis, and twelve others had protocol violations. Of those remaining, 90 patients received amnioinfusions containing cefazolin, and 93 received normal saline placebo. No untoward effects of amnioinfusion were noted in either group.

Groups were similar with respect to demographic variables and with respect to labor and delivery characteristics known to be associated with chorioamnionitis and endometritis (Table 1). Since all patients received amnioinfusions, all had intrauterine pressure catheters.

Analysis of the primary outcome variables demonstrated similar rates of clinical chorioamnionitis and endometritis in the cefazolin and placebo groups (Table 2). The rates of aggregate maternal infection were also similar between the groups. Note that the denominators for endometritis are smaller than for clinical chorioamnionitis. This difference is due to the fact that we did not consider a patient who had clinical chorioamnionitis and then experienced persistent fevers postpartum to also have endometritis. Differences between groups in the rates of suspected and confirmed neonatal infection were also not statistically significant (Table 2). The two cases of confirmed neonatal infection were infants with positive blood cul-
TABLE 2. Outcome variables ${ }^{\mathrm{a}}$

\begin{tabular}{|c|c|c|}
\hline & $\begin{array}{c}\text { Cefazolin } \\
\mathrm{n}=90\end{array}$ & $\begin{array}{c}\text { Placebo } \\
n=93\end{array}$ \\
\hline Chorioamnionitis & $7(7.8,5.0-10.6)$ & $8(8.6,5.7-11.5)$ \\
\hline Endometritis $^{\mathrm{b}}$ & $2(2.4,0.7-4.1)$ & $3(3.5,1.5-5.5)$ \\
\hline Aggregate maternal & & \\
\hline infection & $9(10.0,6.8-13.2)$ & $11(11.8,8.5-15.1)$ \\
\hline $\begin{array}{l}\text { Suspected neonatal } \\
\text { infection }\end{array}$ & $16(17.8,13.8-21.8)$ & $20(21.5,17.2-25.8)$ \\
\hline $\begin{array}{l}\text { Proven neonatal } \\
\text { infection }\end{array}$ & $0(0.0,0.0-3.3)$ & $2(2.2,0.7-3.7)$ \\
\hline
\end{tabular}

${ }^{\text {aD }}$ ata are presented as $\mathrm{n}(\%, 95 \%$ confidence interval). None of the observed differences were statistically significant.

$b_{n}=83$ for the cefazolin group, and $n=85$ for the saline group.

tures. No other neonatal infections (e.g., meningitis, pneumonia) were noted in either group.

\section{DISCUSSION}

In this study, prophylactic addition of cefazolin to amnioinfusions in pregnancies complicated by MSAF did not significantly reduce the rates of clinical chorioamnionitis, endometritis, or neonatal infection. The procedure itself was quite safe, however. The aggregate maternal infection rate (clinical chorioamnionitis or endometritis) in the control group was one third that expected. This effect was due primarily to an extraordinarily low rate of puerperal endometritis. Although some patients with endometritis may have become symptomatic after discharge, none returned to our hospital in this manner, and a large number of such cases is unlikely.

Although not significantly different, there was a slightly lower percentage of infection in the cefazolin group. Nevertheless, given the aggregate infection rate in the control group during the study period, a sample size of approximately $1,400 \mathrm{pa}-$ tients would be required to show a $50 \%$ reduction in the infection rate, assuming an alpha level of 0.05 and a beta of 0.20 . Therefore, a type-II error cannot be excluded. It seems unlikely that such a difference would be clinically significant, however, and prophylactic antibiotics are rarely recommended in situations in which the background rate of infection does not significantly exceed $10 \% .{ }^{19}$

Several factors may explain the lower than anticipated background infection rate. First, since patients undergoing cesarean delivery who were at highest risk for endometritis received a second dose of intravenous antibiotic prophylaxis, the en- 
dometritis rate may have been decreased. Second, we currently employ the universal screening strategy for prevention of early onset neonatal group $B$ streptococcal infection endorsed by the Centers for Disease Control and Prevention. A recent report from our institution showed that rates of clinical chorioamnionitis and endometritis were significantly lower with this approach, as opposed to previously employed strategies. ${ }^{20}$ It is likely that a similar trend is present in patients with MSAF. Finally, during this study period, we screened all pregnant patients for bacterial vaginosis in the late second trimester and treated those women who were infected. Such intervention may well serve to lower the overall rates of maternal infection in our population.

In order to test this intervention as it would be employed clinically, we made no attempt to control for the volume of amnioinfusion patients received. Because many patients certainly delivered before receiving the entire liter of amnioinfusion and some of the antibiotic remains in suspension and flows back out of the cervix, the dose of cefazolin delivered was likely less than one gram, and a larger amount of cefazolin in the suspension may have been more efficacious.

As stated previously, intravenous ampicillinsulbactam given prophylactically in this setting has been shown to significantly reduce the rate of clinical chorioamnionitis. ${ }^{15}$ However, utilization of more narrow spectrum agents for prophylaxis seems prudent, since broad-spectrum agents used in this manner may select for resistant and more virulent strains of microorganisms.

Cefazolin added prophylactically to amnioinfusions administered for MSAF did not significantly reduce the rates of maternal or neonatal infection in this study. Further investigation is needed to elucidate the appropriate role, if any, for antibiotic prophylaxis in patients with this condition.

\section{ACKNOWLEDGMENTS}

The authors thank the resident physicians at the University of Florida/Shands Hospital for their help in enrolling patients, the nurses of the hospital's Labor and Delivery Unit for administering the study amnioinfusions, and Rhonda Cooper, PharmD, research pharmacist.

\section{REFERENCES}

1. Woods JR, Glantz JC. Significance of amniotic fluid meconium. In Creasy RK, Resnik R (eds): Maternal Fetal Medicine: Principles and Practice. 3rd ed. Philadelphia: WB Saunders, 1994, p 413-422.

2. Spong CY, Ogundipe OA, Ross MG. Prophylactic amnioinfusion for meconium-stained amniotic fluid. Am J Obstet Gynecol 1994;171:931-935.

3. Cialone PR, Sherer DM, Ryan RM, Sinkin RA, Abramowicz JS. Amnioinfusion during labor complicated by particulate meconium-stained amniotic fluid decreases neonatal morbidity. Am J Obstet Gynecol 1994;170:842-849.

4. Sadovsky Y, Amon E, Bade ME, Petrie RH. Prophylactic amnioinfusion during labor complicated by meconium: a preliminary report. Am J Obstet Gynecol 1989; 161:613-617.

5. Eriksen NL, Hostetter M, Parisi VM. Prophylactic amnioinfusion in pregnancies complicated by thick meconium. Am J Obstet Gynecol 1994;171:1026-1030.

6. Macri CJ, Schrimmer DB, Leung A, Greenspoon JS, Paul RH. Prophylactic amnioinfusion improves outcome of pregnancy complicated by thick meconium and oligohydramnios. Am J Obstet Gynecol 1992;167:117121.

7. Wenstrom KD, Parsons MT. The prevention of meconium aspiration in labor using amnioinfusion. Obstet Gynecol 1989;73:647-651.

8. Dye T, Aubry R, Gross S, Artal R. Amnioinfusion and the intrauterine prevention of meconium aspiration. Am J Obstet Gynecol 1994;171:1601-1605.

9. Hoskins IA, Hemming VG, Johnson TRB, Winkel CA. Effects of alterations of zinc-to-phosphorus ratios and meconium content on group B streptococcus growth in human amniotic fluid in vitro. Am J Obstet Gynecol 1987;157:770-773.

10. Florman AL, Teubner D. Enhancement of bacterial growth in amniotic fluid by meconium. J Pediatr 1969; 74:111-114.

11. Clark $P$, Duff $P$. Inhibition of neutrophil oxidative burst and phagocytosis by meconium. Am J Obstet Gynecol 1995;173:1301-1305.

12. Chapman S, Duff P. Incidence of chorioamnionitis in patients with meconium-stained amniotic fluid. Infect Dis Obstet Gynecol 1995;2:210-212.

13. Wen TS, Eriksen NL, Blanco JD, Graham JM, Oshiro BT, Prieto JA. Association of clinical intra-amniotic infection and meconium. Am J Perinatol 1993;10:438-440.

14. Markovitch O, Mazor M, Shoham-Vardi I, Chaim W, Leiberman JR, Glezerman M. Meconium stained amniotic fluid is associated with maternal infectious morbidity in preterm delivery. Acta Obstet Gynecol Scand 1993;72:538-542.

15. Adair CD, Ernest JM, Sanchez-Ramos L, Burrus DR, Boles ML, Veille JC. Meconium-stained amniotic fluidassociated infectious morbidity: a randomized, double- 
blind trial of ampicillin-sulbactam prophylaxis. Obstet Gynecol 1996;88:216-220.

16. Mazor M, Furman B, Wiznitzer A, Shoham-Vardi I, Cohen J, Ghezzi F. Maternal and perinatal outcome of patients with preterm labor and meconium-stained amniotic fluid. Obstet Gynecol 1995;86:830-833.

17. Piper JM, Newton ER, Berkus MD, Peairs WA. Meconium: a marker for peripartum infection. Obstet Gynecol 1998;91:741-745.

18. Usta IM, Mercer BM, Aswad NK, Sibai BM. The im- pact of a policy of amnioinfusion for meconium-stained amniotic fluid. Obstet Gynecol 1995;85:237-241.

19. Duff P, Smith PN, Keiser JF. Antibiotic prophylaxis in low risk cesarean section. J Reprod Med 1982;27:133138.

20. Locksmith GJ, Clark P, Duff P. Maternal and neonatal infection rates with three different protocols for prevention of group B streptococcal disease. Am J Obstet Gynecol 1999;180:416-422. 


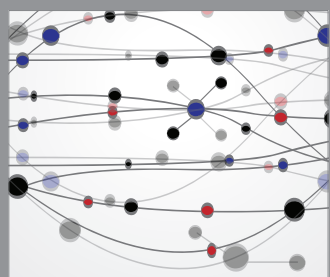

The Scientific World Journal
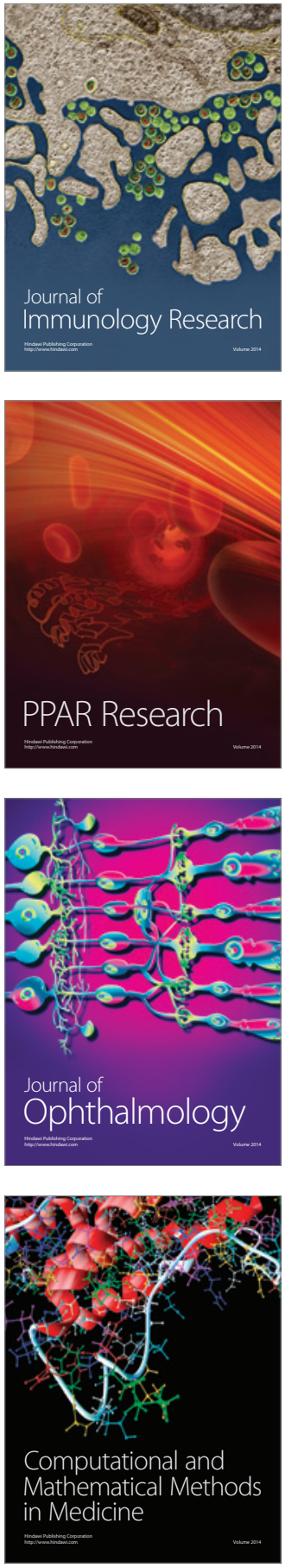

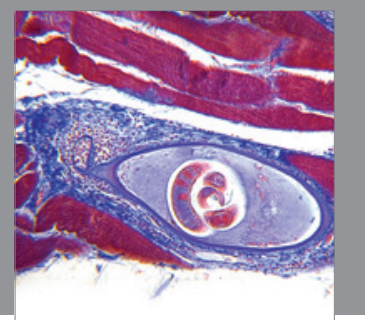

Gastroenterology

Research and Practice
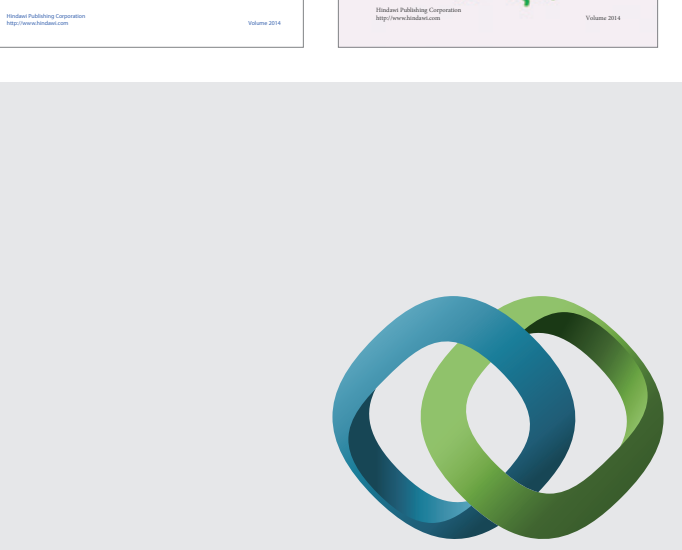

\section{Hindawi}

Submit your manuscripts at

http://www.hindawi.com
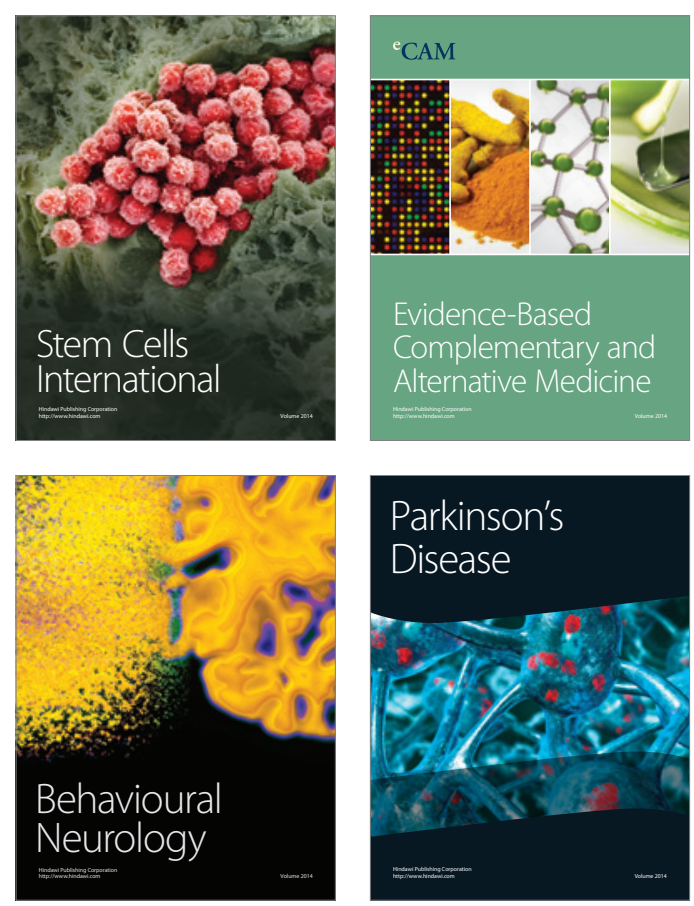

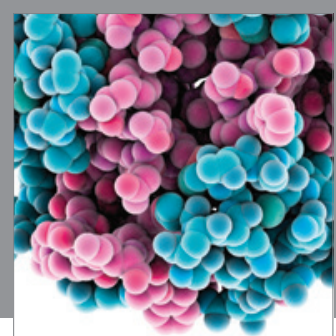

Journal of
Diabetes Research

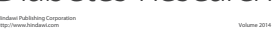

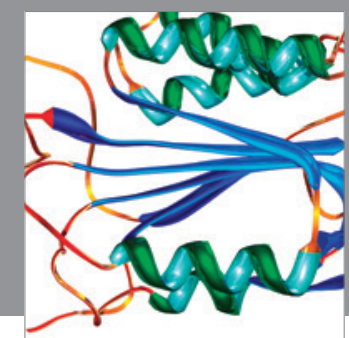

Disease Markers
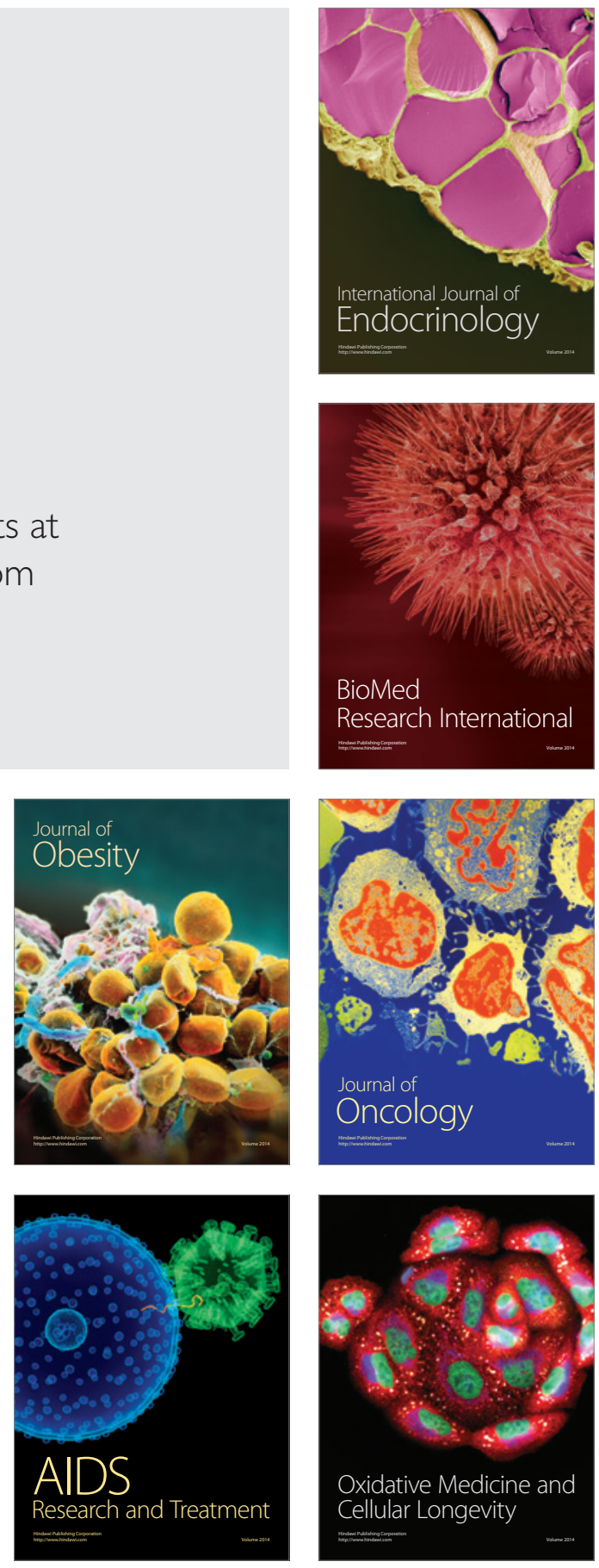